\title{
Stabilisation of Hybrid Stochastic Differential Equations by Delay Feedback Control
}

\author{
Xuerong Mao ${ }^{1}$, James Lam ${ }^{2}$, Lirong Huang ${ }^{1}$ \\ ${ }^{1}$ Department of Statistics and Modelling Science, \\ University of Strathclyde, Glasgow G1 1XH, U.K. \\ 2 Department of Mechanical Engineering, \\ University of Hong Kong, Hong Kong
}

\begin{abstract}
This paper is concerned with the exponential mean-square stabilisation of hybrid stochastic differential equations (also known as stochastic differential equations with Markovian switching) by delay feedback controls. Although the stabilisation by nondelay feedback controls for such equations has been discussed by several authors, there is so far little on the stabilisation by delay feedback controls and our aim here is mainly to close the gap. To make our theory more understandable as well as to avoid complicated notations, we will restrict our underlying hybrid stochastic differential equations to a relatively simple form. However our theory can certainly be developed to cope with much more general equations without any difficulty.
\end{abstract}

Key words: Brownian motion, Markov chain, exponential mean-square stability, linear Matrix inequality.

\section{Introduction}

The hybrid systems driven by continuous-time Markov chains have been used to model many practical systems where they may experience abrupt changes in their structure and parameters. The hybrid systems combine a part of the state that takes values continuously and another part of the state that takes discrete values. An important class of hybrid systems is the hybrid stochastic differential equation (SDE),

$$
d x(t)=f(x(t), r(t), t) d t+g(x(t), r(t), t) d w(t),
$$

where a part of the state $x(t)$ takes values in $R^{n}$ while another part of the state $r(t)$ is a Markov chain taking values in a finite space $S=\{1,2, \cdots, N\}$. Such an SDE is also known as the SDE with Markovian switching. One of the important issues in the study of hybrid SDEs is the automatic control, with subsequent emphasis being placed on the analysis of stability. There is an intensive literature in this area, for example, $[1,8,14,15,16,18,19,20,22,25,26,27,28]$, a few to name. In particular, we refer the reader to the recent book [21]. 
This paper is concerned with the exponential mean-square stabilisation of hybrid stochastic differential equations (also known as stochastic differential equations with Markovian switching) by delay feedback controls. The stabilisation by non-delay feedback controls for such equations has been discussed by several authors e.g. [20]. Here, given an unstable hybrid SDE in the form of (1.1), it is required to find a feedback control $u(x(t), r(t))$, based on the current state, so that the controlled system

$$
d x(t)=[f(x(t), r(t), t)+u(x(t), r(t))] d t+g(x(t), r(t), t) d w(t)
$$

becomes stable. (It is possible to put the feedback control in the diffusion part but, in this paper, we will only consider the case where the control is put into the drift part.) However, it is more realistic in practice if the control depends on a past state, say $x(t-\tau)$, due to a time lag $\tau(>0)$ between the time when the observation of the state is made and the time when the feedback control reaches the system. Accordingly, the control should be of the form $u(x(t-\tau), r(t))$. Hence, the stabilisation problem becomes to design a delay feedback control $u(x(t-\tau), r(t))$ in the drift part so that the controlled system

$$
d x(t)=[f(x(t), r(t), t)+u(x(t-\tau), r(t))] d t+g(x(t), r(t), t) d w(t)
$$

becomes stable. There is so far little on this stabilisation problem by delay feedback controls and our aim here is mainly to close the gap.

Of course, one may consider to design a feedback control $u(x(t), x(t-\tau), r(t))$, based on both current and past state, so that the controlled system

$$
d x(t)=[f(x(t), r(t), t)+u(x(t), x(t-\tau), r(t))] d t+g(x(t), r(t), t) d w(t)
$$

becomes stable. However, this is clearly easier than either (1.2) or (1.3) because (1.4) is possible if either (1.2) or (1.3) is possible.

To make our theory more understandable as well as to avoid complicated notations, we will restrict our underlying hybrid systems to a relatively simple form. However our theory can certainly be developed to cope with much more general equations without any difficulty. For example, the underlying system would be an unstable hybrid stochastic differential delay equation

$$
d x(t)=f(x(t), x(t-\delta), r(t), t) d t+g(x(t), x(t-\delta), r(t), t) d w(t),
$$

and we could show that it is possible to design a delay feedback control $u(x(t-\tau), r(t))$ in the drift part so that the controlled system

$$
d x(t)=[f(x(t), x(t-\delta), r(t), t)+u(x(t-\tau), r(t))] d t+g(x(t), x(t-\delta), r(t), t) d w(t)
$$

becomes stable.

\section{Notation and Stabilisation Problem}

Throughout this paper, unless otherwise specified, we let $\left(\Omega, \mathcal{F},\left\{\mathcal{F}_{t}\right\}_{t \geq 0}, \mathbb{P}\right)$ be a complete probability space with a filtration $\left\{\mathcal{F}_{t}\right\}_{t \geq 0}$ satisfying the usual conditions (i.e. it is right continuous and $\mathcal{F}_{0}$ contains all $P$-null sets). Let $w(t)$ be a scalar Brownian motion defined on the probability space. If $A$ is a vector or matrix, its transpose is denoted by $A^{T}$. If $A$ 
is a matrix, its operator norm is denoted by $\|A\|=\sup \{|A x|:|x|=1\}$, where $|\cdot|$ is the Euclidean norm.

Let $r(t), t \geq 0$, be a right-continuous Markov chain on the probability space taking values in a finite state space $S=\{1,2, \cdots, N\}$ with generator $\Gamma=\left(\gamma_{i j}\right)_{N \times N}$ given by

$$
\mathbb{P}\{r(t+\Delta)=j \mid r(t)=i\}= \begin{cases}\gamma_{i j} \Delta+o(\Delta) & \text { if } i \neq j, \\ 1+\gamma_{i i} \Delta+o(\Delta) & \text { if } i=j,\end{cases}
$$

where $\Delta>0$. Here $\gamma_{i j} \geq 0$ is the transition rate from $i$ to $j$ if $i \neq j$ while

$$
\gamma_{i i}=-\sum_{j \neq i} \gamma_{i j}
$$

We assume that the Markov chain $r(\cdot)$ is independent of the Brownian motion $w(\cdot)$. It is known that almost all sample paths of $r(t)$ are constant except for a finite number of simple jumps in any finite subinterval of $R_{+}(:=[0, \infty))$. We stress that almost all sample paths of $r(t)$ are right continuous.

Consider an $n$-dimensional linear hybrid SDE

$$
d x(t)=A(r(t)) x(t) d t+B(r(t)) x(t) d w(t)
$$

on $t \geq 0$. Here $A, B: S \rightarrow R^{n \times n}$ and we will often write $A(i)=A_{i}$ and $B(i)=B_{i}$. Suppose that this given equation is unstable and we are required to design a feedback control $u(x(t), r(t))$ in the drift part so that the controlled SDE

$$
d x(t)=[A(r(t)) x(t)+u(x(t), r(t))] d t+B(r(t)) x(t) d w(t)
$$

will be mean-square exponentially stable, where $u$ is a mapping from $R^{n} \times S$ to $R^{n}$. We here note that the feedback control $u(x(t), r(t))$ depends on the current state $x(t)$. However, it is more realistic in practice if the control depends on a past state, say $x(t-\tau)$, due to a time lag $\tau(>0)$ between the time when the observation of the state is made and the time when the feedback control reaches the system. Accordingly, the control should be of the form $u(x(t-\tau), r(t))$. The stabilisation problem hence becomes to design a delay feedback control $u(x(t-\tau), r(t))$ in the drift part so that the controlled system

$$
d x(t)=[A(r(t)) x(t)+u(x(t-\tau), r(t))] d t+B(r(t)) x(t) d w(t)
$$

will be mean-square exponentially stable. As the given SDE (2.1) is linear, it is natural to use a linear feedback control. One of the most common linear feedback controls is the structure control of the form $u(x, i)=F(i) G(i) x$, where $F$ and $G$ are mappings from $S$ to $R^{n \times l}$ and $R^{l \times n}$, respectively, and one of them is given while the other needs to be designed. These two cases are known as:

- State feedback: design $F(\cdot)$ when $G(\cdot)$ is given;

- Output injection: design $G(\cdot)$ when $F(\cdot)$ is given.

Again, we will often write $F(i)=F_{i}$ and $G(i)=G_{i}$. As a result, the controlled system (2.2) becomes

$$
d x(t)=[A(r(t)) x(t)+F(r(t)) G(r(t)) x(t-\tau)] d t+B(r(t)) x(t) d w(t) .
$$


This controlled system is a hybrid stochastic differential delay equation (SDDE). For an SDDE, it is required to know some initial data, for example, $x(t)$ on $t \in[-\tau, 0]$ in order for its solution to be well defined. Given that our underlying equation (2.1) is non-delay and it only requires the initial value $x(0) \in R^{n}$, it is more natural to assume that for our controlled system (2.3) we know the initial data $x(t)$ on $t \in[0, \tau]$. This can be interpreted as follows: Let the underlying equation (2.1) evolve from time 0 to $\tau$ and observe the whole segment $\{x(t): 0 \leq t \leq \tau\}$. Starting from $\tau$ on, design the feedback control $F(r(t)) G(r(t)) x(t-\tau)$ based on the past observation $\{x(t): 0 \leq t \leq \tau\}$ as well as furthermore observation as time evolves. In other words, we shall regard the controlled system (2.3) as an SDDE on $t \geq \tau$ with the initial data $\{x(t): 0 \leq t \leq \tau\}$ which are generated by the $\operatorname{SDE}(2.1)$ given the initial value $x(0) \in R^{n}$. By the theory of hybrid SDEs (see e.g. [14]), we know

$$
\mathbb{E}|x(t)|^{2}<\infty \quad \text { on } t \in[0, \tau]
$$

which in turn implies, by the theory of hybrid SDDEs (see e.g. [21]), that

$$
\mathbb{E}|x(t)|^{2}<\infty \quad \text { for } t \geq \tau .
$$

Our aim is to design either $G(\cdot)$ given $F(\cdot)$ or $F(\cdot)$ given $G(\cdot)$ so that $\mathbb{E}|x(t)|^{2}$ will tend to zero exponentially. We shall discuss the former case in the next section while leave the later case to Section 4.

\section{State Feedback: Design $F(\cdot)$ when $G(\cdot)$ is given}

One technique used frequently in the study of stability of SDDEs is the method of linear matrix inequalities (LMIs) (see e.g. [4, 5, 7, 24, 29, 30, 31, 32]), although there are other methods (see e.g. the recent survey paper [17]). The principal procedure of the LMI method is: (i) Design a positive-definite quadratic Lyapunov function or functional $V$. (ii) Apply the Itô formula to compute the Itô differential $d V$. (iii) Arrange the drift part of $d V$ in the form of LMIs. For our stabilisation purpose related to the controlled SDDE (2.3) we shall use a positive-definite quadratic Lyapunov functional on the segment $\hat{x}_{t}:=\{x(t+s):-2 \tau \leq s \leq 0\}$ for $t \geq 2 \tau$. More precisely, the Lyapunov functional used in this paper will be of the form

$$
V\left(\hat{x}_{t}, r(t), t\right)=x^{T}(t) Q(r(t)) x(t)+\int_{t-\tau}^{t} \int_{s}^{t}\left[\alpha_{1}|x(u)|^{2}+\alpha_{2}|x(u-\tau)|^{2}\right] d u d s
$$

for $t \geq 2 \tau$. Here $\alpha_{1}$ and $\alpha_{2}$ are two positive numbers while $Q$ is defined on $S$ and takes its values of symmetric positive-definite $n \times n$-matrices. Of course, we shall write $Q(i)=Q_{i}$. Accordingly we shall regard the controlled system (2.3) as an SDDE on $t \geq 2 \tau$ with initial data $\{x(s): 0 \leq s \leq 2 \tau\}$. Applying the Itô formula (see e.g. [16, 21]) to the Lyapunov functional defined by (3.1) yields

$$
d V\left(\hat{x}_{t}, r(t), t\right)=L V\left(\hat{x}_{t}, r(t), t\right) d t+2 x^{T}(t) Q(r(t)) B(r(t)) x(t) d w(t)
$$


for $t \geq 2 \tau$, where, when $r(t)=i$,

$$
\begin{aligned}
L V\left(\hat{x}_{t}, i, t\right) & =2 x^{T}(t) Q_{i}\left[A_{i} x(t)+F_{i} G_{i} x(t-\tau)\right] \\
& +x^{T}(t) B_{i} Q_{i} B_{i} x(t)+\sum_{j=1}^{N} \gamma_{i j} x^{T}(t) Q_{j} x(t) \\
& +\alpha_{1} \tau|x(t)|^{2}-\alpha_{1} \int_{t-\tau}^{t}|x(s)|^{2} d s \\
& +\alpha_{2} \tau|x(t-\tau)|^{2}-\alpha_{2} \int_{t-\tau}^{t}|x(s-\tau)|^{2} d s .
\end{aligned}
$$

To see why (3.2) holds, we regard the solution $x(t)$ of equation (2.3) as an Itô process and apply the Itô formula (see e.g. $[16,21])$ to $x^{T}(t) Q(r(t)) x(t)$ to get

$$
\begin{aligned}
d\left[x^{T}(t) Q(r(t)) x(t)\right]= & \left(2 x^{T}(t) Q(r(t))[A(r(t)) x(t)+F(r(t)) G(r(t)) x(t-\tau)]\right. \\
& \left.\quad+x^{T}(t) B(r(t)) Q(r(t)) B(r(t)) x(t)+\sum_{j=1}^{N} \gamma_{r(t), j} x^{T}(t) Q_{j} x(t)\right) d t \\
+ & 2 x^{T}(t) Q(r(t)) B(r(t)) x(t) d w(t) .
\end{aligned}
$$

On the other hand, the fundamental theory of calculus shows

$$
\begin{aligned}
& d\left(\int_{t-\tau}^{t} \int_{s}^{t}\left[\alpha_{1}|x(u)|^{2}+\alpha_{2}|x(u-\tau)|^{2}\right] d u d s\right) \\
= & \left(\alpha_{1} \tau|x(t)|^{2}-\alpha_{1} \int_{t-\tau}^{t}|x(s)|^{2} d s+\alpha_{2} \tau|x(t-\tau)|^{2}-\alpha_{2} \int_{t-\tau}^{t}|x(s-\tau)|^{2} d s\right) d t .
\end{aligned}
$$

Combining these two equalities gives (3.2). Let us now present a useful lemma.

Lemma 3.1 If there are numbers $\lambda_{1}>\lambda_{2} \geq 0$ and $\lambda_{3}>0$ such that

$$
\mathbb{E}\left(L V\left(\hat{x}_{t}, r(t), t\right)\right) \leq-\lambda_{1} \mathbb{E}|x(t)|^{2}+\lambda_{2} \mathbb{E}|x(t-\tau)|^{2}-\lambda_{3} \mathbb{E} \int_{t-2 \tau}^{t}|x(s)|^{2} d s
$$

for all $t \geq 2 \tau$, then

$$
\limsup _{t \rightarrow \infty} \frac{1}{t} \log \left(\mathbb{E}|x(t)|^{2}\right) \leq-\gamma
$$

where $\gamma>0$ obeys

$$
\gamma \leq \frac{\lambda_{3}}{\tau\left(\alpha_{1} \vee \alpha_{2}\right)} \quad \text { and } \quad \lambda_{1} \geq \lambda_{2} e^{\gamma \tau}+\gamma \check{q}
$$

in which $\check{q}=\max _{i \in S}\left\|Q_{i}\right\|$.

Proof. By the Itô formula (see e.g. [16, 21]), we have

$$
e^{\gamma t} \mathbb{E} V\left(\hat{x}_{t}, r(t), t\right)=C+\int_{2 \tau}^{t} e^{\gamma s}\left[\gamma \mathbb{E} V\left(\hat{x}_{s}, r(s), s\right)+\mathbb{E}\left(L V\left(\hat{x}_{s}, r(s), s\right)\right)\right] d s,
$$


where $C=e^{2 \gamma \tau} \mathbb{E} V\left(\hat{x}_{2 \tau}, r(2 \tau), 2 \tau\right)$. We compute

$$
\begin{aligned}
\mathbb{E} V\left(\hat{x}_{t}, r(t), t\right) & \leq \check{q} \mathbb{E}|x(t)|^{2}+\int_{t-\tau}^{t} \int_{s}^{t}\left[\alpha_{1} \mathbb{E}|x(u)|^{2}+\alpha_{2} \mathbb{E}|x(u-\tau)|^{2}\right] d u d s \\
& \leq \check{q} \mathbb{E}|x(t)|^{2}+\int_{t-\tau}^{t}\left[\alpha_{1} \tau \mathbb{E}|x(u)|^{2}+\alpha_{2} \tau \mathbb{E}|x(u-\tau)|^{2}\right] d u \\
& \leq \check{q} \mathbb{E}|x(t)|^{2}+\tau\left(\alpha_{1} \vee \alpha_{2}\right) \int_{t-2 \tau}^{t} \mathbb{E}|x(u)|^{2} d u .
\end{aligned}
$$

Substituting this and (3.4) into (3.6) and noting that $\lambda_{3} \geq \tau \gamma\left(\alpha_{1} \vee \alpha_{2}\right)$, we get

$$
e^{\gamma t} \mathbb{E} V\left(\hat{x}_{t}, r(t), t\right) \leq C+\int_{2 \tau}^{t} e^{\gamma s}\left[\left(-\lambda_{1}+\gamma \check{q}\right) \mathbb{E}|x(s)|^{2}+\lambda_{2} \mathbb{E}|x(s-\tau)|^{2}\right] d s .
$$

But

$$
\begin{aligned}
& \int_{2 \tau}^{t} e^{\gamma s} \mathbb{E}|x(s-\tau)|^{2} d s=\int_{\tau}^{t-\tau} e^{\gamma(s+\tau)} \mathbb{E}|x(s)|^{2} d s \\
& \leq \int_{\tau}^{2 \tau} e^{\gamma(s+\tau)} \mathbb{E}|x(s)|^{2} d s+\int_{\tau}^{t} e^{\gamma(s+\tau)} \mathbb{E}|x(s)|^{2} d s .
\end{aligned}
$$

Hence, recalling that $\lambda_{1}-\gamma \check{q} \geq \lambda_{2} e^{\gamma \tau}$,

$$
e^{\gamma t} \mathbb{E} V\left(\hat{x}_{t}, r(t), t\right) \leq C+\lambda_{2} \int_{\tau}^{2 \tau} e^{\gamma(s+\tau)} \mathbb{E}|x(s)|^{2} d s .
$$

But we clearly have

$$
\mathbb{E} V\left(\hat{x}_{t}, r(t), t\right) \geq \hat{q} \mathbb{E}|x(t)|^{2},
$$

where $\hat{q}=\min _{i \in S} \lambda_{\min }\left(Q_{i}\right)>0$. We therefore obtain

$$
\hat{q} e^{\gamma t} \mathbb{E}|x(t)|^{2} \leq C+\lambda_{2} \int_{\tau}^{2 \tau} e^{\gamma s+\tau} \mathbb{E}|x(s)|^{2} d s,
$$

which implies the desired assertion (3.5) immediately.

Theorem 3.2 Choose five positive numbers $\alpha_{1}, \alpha_{2}, \alpha_{3}$ and $\beta_{1}, \beta_{2}$ such that

$$
\beta_{1}>\max _{i \in S}\left(2\left\|B_{i}\right\|^{2}\right), \quad \alpha_{1}>\alpha_{3} \beta_{1}, \quad \alpha_{2}>\alpha_{3} \beta_{2} .
$$

Assume that for these chosen numbers, the following LMIs

$$
\left[\begin{array}{cc}
\bar{K}_{i} & Y_{i} G_{i} \\
G_{i}^{T} Y_{i}^{T} & -\alpha_{3} I
\end{array}\right]<0, \quad i \in S
$$

have solutions $\bar{\tau}>0$ and $Q_{i}, Y_{i} \in R^{n \times n}$ with $Q_{i}=Q_{i}^{T}>0$, where $I$ is the $n \times n$ identity matrix and

$$
\bar{K}_{i}=Q_{i} A_{i}+Y_{i} G_{i}+A_{i}^{T} Q_{i}+G_{i}^{T} Y_{i}+B_{i}^{T} Q_{i} B_{i}+\sum_{j=1}^{N} \gamma_{i j} Q_{j}+\left(\alpha_{1}+\alpha_{2}\right) \bar{\tau} I .
$$

Let $\tau^{*}$ be the largest number in $(0, \bar{\tau}]$ which obeys

$$
\max _{i \in S}\left(4 \tau^{*}\left\|A_{i}\right\|^{2}+2\left\|B_{i}\right\|^{2}\right) \leq \beta_{1} \quad \text { and } \quad \max _{i \in S}\left(4 \tau^{*}\left\|Q_{i}^{-1} Y_{i} G_{i}\right\|^{2}\right) \leq \beta_{2} .
$$

Then, if $\tau \leq \tau^{*}$, by setting

$$
F_{i}=Q_{i}^{-1} Y_{i}, \quad i \in S,
$$

the controlled system (2.3) is exponentially stable in mean square. 
Proof. We first note that by the well-known Schur complements, the LMIs (3.8) are equivalent to the following matrix inequalities (MIs)

$$
\bar{H}_{i}:=\bar{K}_{i}+\alpha_{3}^{-1} Y_{i} G_{i}\left(Y_{i} G_{i}\right)^{T}<0, \quad i \in S .
$$

With $F_{i}$ defined by (3.10), we compute

$$
\begin{aligned}
& 2 x^{T}(t) Q_{i}\left[A_{i} x(t)+F_{i} G_{i} x(t-\tau)\right] \\
= & 2 x^{T}(t) Q_{i}\left[\left(A_{i}+F_{i} G_{i}\right) x(t)-F_{i} G_{i}(x(t)-x(t-\tau))\right] \\
\leq & x^{T}(t)\left[Q_{i}\left(A_{i}+F_{i} G_{i}\right)+\left(A_{i}+F_{i} G_{i}\right)^{T} Q_{i}\right] x(t) \\
+ & \alpha_{3}^{-1}\left|x^{T}(t) Q_{i} F_{i} G_{i}\right|^{2}+\alpha_{3}|x(t)-x(t-\tau)|^{2} .
\end{aligned}
$$

Hence, we see from (3.3) that

$$
\begin{aligned}
L V\left(\hat{x}_{t}, i, t\right) & \leq x^{T}(t) H_{i} x(t)-\alpha_{2} \tau\left(|x(t)|^{2}-|x(t-\tau)|^{2}\right)+\alpha_{3}|x(t)-x(t-\tau)|^{2} \\
& -\alpha_{1} \int_{t-\tau}^{t}|x(s)|^{2} d s-\alpha_{2} \int_{t-\tau}^{t}|x(s-\tau)|^{2} d s
\end{aligned}
$$

where

$$
H_{i}=K_{i}+\alpha_{3}^{-1} Y_{i} G_{i}\left(Y_{i} G_{i}\right)^{T},
$$

in which

$$
K_{i}=Q_{i} A_{i}+Y_{i} G_{i}+A_{i}^{T} Q_{i}+G_{i}^{T} Y_{i}+B_{i}^{T} Q_{i} B_{i}+\sum_{j=1}^{N} \gamma_{i j} Q_{j}+\left(\alpha_{1}+\alpha_{2}\right) \tau I .
$$

As $\tau \leq \bar{\tau}$, we see from (3.11) that

$$
H_{i}<0, \quad i \in S
$$

Noting

$$
\begin{aligned}
x(t)-x(t-\tau) & =\int_{t-\tau}^{t}[A(r(s)) x(s)+F(r(s)) G(r(s)) x(s-\tau)] d s \\
& +\int_{t-\tau}^{t} B(r(s)) x(s) d w(s),
\end{aligned}
$$

we estimate

$$
\begin{aligned}
\mathbb{E}|x(t)-x(t-\tau)|^{2} & \leq 2 \tau \mathbb{E} \int_{t-\tau}^{t}|A(r(s)) x(s)+F(r(s)) G(r(s)) x(s-\tau)|^{2} d s \\
& +2 \mathbb{E} \int_{t-\tau}^{t}|B(r(s)) x(s)|^{2} d s \\
& \leq \bar{\beta}_{1} \mathbb{E} \int_{t-\tau}^{t}|x(s)|^{2} d s+\bar{\beta}_{2} \mathbb{E} \int_{t-\tau}^{t}|x(s-\tau)|^{2} d s,
\end{aligned}
$$

where

$$
\bar{\beta}_{1}=\max _{i \in S}\left(4 \tau\left\|A_{i}\right\|^{2}+2\left\|B_{i}\right\|^{2}\right) \quad \text { and } \quad \bar{\beta}_{2}=\max _{i \in S}\left(4 \tau\left\|F_{i} G_{i}\right\|^{2}\right) \text {. }
$$

Recalling (3.9) we observe that $\bar{\beta}_{1} \leq \beta_{1}$ and $\bar{\beta}_{2} \leq \beta_{2}$. Set

$$
-\lambda=\max _{i \in S} \lambda_{\max }\left(H_{i}\right) .
$$


By (3.13), $\lambda>0$. Now, replacing $i$ by $r(t)$ in (3.12), taking the expectation on both sides of (3.12) and then making use the above estimations we obtain

$$
\begin{aligned}
\mathbb{E}\left(L V\left(\hat{x}_{t}, r(t), t\right)\right) & \leq-\left(\lambda+\alpha_{2} \tau\right) \mathbb{E}|x(t)|^{2}+\alpha_{2} \tau \mathbb{E}|x(t-\tau)|^{2} \\
& -\left(\alpha_{1}-\alpha_{3} \beta_{1}\right) \mathbb{E} \int_{t-\tau}^{t}|x(s)|^{2} d s \\
& -\left(\alpha_{2}-\alpha_{3} \beta_{2}\right) \mathbb{E} \int_{t-\tau}^{t}|x(s-\tau)|^{2} d s \\
& \leq-\lambda_{1} \mathbb{E}|x(t)|^{2}+\lambda_{2} \mathbb{E}|x(t-\tau)|^{2}-\lambda_{3} \mathbb{E} \int_{t-2 \tau}^{t}|x(s)|^{2} d s,
\end{aligned}
$$

where

$$
\lambda_{1}:=\left(\lambda+\alpha_{2} \tau\right)>\lambda_{2}:=\alpha_{2} \tau \quad \text { and } \quad \lambda_{3}:=\left(\alpha_{1}-\alpha_{3} \beta_{1}\right) \wedge\left(\alpha_{1}-\alpha_{3} \beta_{1}\right)>0 .
$$

Hence the conclusion follows from Lemma 3.1.

\section{Output Injection: Design $G(\cdot)$ when $F(\cdot)$ is given}

Let us now discuss the case where we are given the mapping $F: S \rightarrow R^{n \times l}$ but are required to design the mapping $G: S \rightarrow R^{l \times n}$. The following theorem gives an answer.

Theorem 4.1 Choose five positive numbers $\alpha_{1}, \alpha_{2}, \alpha_{3}$ and $\beta_{1}, \beta_{2}$ such that

$$
\beta_{1}>\max _{i \in S}\left(2\left\|B_{i}\right\|^{2}\right), \quad \alpha_{1}>\alpha_{3} \beta_{1}, \quad \alpha_{2}>\alpha_{3} \beta_{2} .
$$

Choose furthermore $N$ positive numbers $\rho_{i}, i \in S$. Assume that for these chosen numbers, the following LMIs

$$
\left[\begin{array}{cccc}
M_{i 1} & F_{i} Y_{i} & \sqrt{\alpha_{1}+\alpha_{2}} X_{i} & M_{i 2} \\
Y_{i}^{T} F_{i}^{T} & -\alpha_{3} \rho_{i} I & 0 & 0 \\
\sqrt{\alpha_{1}+\alpha_{2}} X_{i} & 0 & -\bar{\tau} I & 0 \\
M_{i 2}^{T} & 0 & 0 & -M_{i 3}
\end{array}\right]<0, \quad i \in S
$$

and

$$
-2 X_{i}+\left(1+\rho_{i}\right) I<0, \quad i \in S
$$

have solutions $\bar{\tau}>0$ and $X_{i}, Y_{i} \in R^{n \times n}$ with $X_{i}=X_{i}^{T}>0$, where $I$ is the $n \times n$ identity matrix and

$$
\begin{gathered}
M_{i 1}=A_{i} X_{i}+F_{i} Y_{i}+X_{i} A_{i}^{T}+Y_{i}^{T} F_{i}^{T}+\gamma_{i i} X_{i}, \\
M_{i 2}=\left[\sqrt{\gamma_{i 1}} X_{i}, \cdots, \sqrt{\gamma_{i(i-1)}} X_{i}, X_{i} B_{i}^{T}, \sqrt{\gamma_{i(i+1)}} X_{i}, \cdots, \sqrt{\gamma_{i N}} X_{i}\right], \\
M_{i 3}=\operatorname{diag}\left(X_{1}, \cdots, X_{N}\right) .
\end{gathered}
$$

Let $\tau^{*}$ be the largest number in $(0, \bar{\tau}]$ which obeys

$$
\max _{i \in S}\left(4 \tau^{*}\left\|A_{i}\right\|^{2}+2\left\|B_{i}\right\|^{2}\right) \leq \beta_{1} \quad \text { and } \quad \max _{i \in S}\left(4 \tau^{*}\left\|F_{i} X_{i}^{-1} Y_{i}\right\|^{2}\right) \leq \beta_{2}
$$

Then, if $\tau \leq \tau^{*}$, by setting

$$
G_{i}=X_{i}^{-1} Y_{i}, \quad i \in S,
$$

the controlled system (2.3) is exponentially stable in mean square. 
Proof. Let $Q_{i}=X_{i}^{-1}$ and $V$ be the same as defined by (3.1). We still have (3.12) with

$$
H_{i}=K_{i}+\alpha_{3}^{-1} Q_{i} F_{i} G_{i} G_{i}^{T} F_{i}^{T} Q_{i}
$$

in which

$$
K_{i}=Q_{i} A_{i}+Q_{i} F_{i} G_{i}+A_{i}^{T} Q_{i}+G_{i}^{T} F_{i}^{T} Q_{i}+B_{i}^{T} Q_{i} B_{i}+\sum_{j=1}^{N} \gamma_{i j} Q_{j}+\left(\alpha_{1}+\alpha_{2}\right) \tau I .
$$

Hence, if we can show that

$$
H_{i}<0, \quad i \in S
$$

we can still imply (3.14) from (3.12) and hence obtain the assertion by Lemma 3.1 in the same way as Theorem 3.2 was proved. In other words, to complete the proof, all we need to do is to show (4.6).

By the Schur complements, (4.6) holds if and only if

$$
\left[\begin{array}{ccc}
U_{i} & Q_{i} F_{i} G_{i} & \sqrt{\alpha_{1}+\alpha_{2}} Q_{i} \\
G_{i}^{T} F_{i}^{T} Q_{i} & -\alpha_{3} I & 0 \\
\sqrt{\alpha_{1}+\alpha_{2}} Q_{i} & 0 & -\tau^{-1} Q_{i}^{2}
\end{array}\right]<0, \quad i \in S
$$

where

$$
U_{i}=Q_{i} A_{i}+Q_{i} F_{i} G_{i}+A_{i}^{T} Q_{i}+G_{i}^{T} F_{i}^{T} Q_{i}+B_{i}^{T} Q_{i} B_{i}+\sum_{j=1}^{N} \gamma_{i j} Q_{j}
$$

Noting that $X_{i}=Q_{i}^{-1}$ and $Y_{i}=G_{i} X_{i}$, we can pre- and post-multiply (4.7) to see that (4.7) is equivalent to

$$
\left[\begin{array}{ccc}
X_{i} U_{i} X_{i} & F_{i} Y_{i} & \sqrt{\alpha_{1}+\alpha_{2}} X_{i} \\
Y_{i}^{T} F_{i}^{T} & -\alpha_{3} X_{i}^{2} & 0 \\
\sqrt{\alpha_{1}+\alpha_{2}} X_{i} & 0 & -\tau^{-1} I
\end{array}\right]<0, \quad i \in S
$$

where

$$
\begin{aligned}
X_{i} U_{i} X_{i}= & A_{i} X_{i}+F_{i} Y_{i}+X_{i} A_{i}^{T}+Y_{i}^{T} F_{i}^{T}+X_{i} B_{i}^{T} X_{i}^{-1} B_{i} X_{i} \\
& +\gamma_{i i} X_{i}+\sum_{j \neq i} \gamma_{i j} X_{i} X_{j}^{-1} X_{i} .
\end{aligned}
$$

Now, noting that

$$
0 \leq\left(X_{i}-\rho_{i} I\right)^{2}=X_{i}^{2}-2 \rho_{i} X_{i}+\rho_{i}^{2} I=X_{i}^{2}+\rho_{i}\left(-2 X_{i}+\rho_{i}\right) I,
$$

and recalling condition (4.3), we observe that

$$
-X_{i}^{2} \leq \rho_{i}\left(-2 X_{i}+\rho_{i}\right) I=\rho_{i}\left[-2 X_{i}+\left(1+\rho_{i}\right) I\right]-\rho_{i} I<-\rho_{i} I .
$$

Also, $\tau \leq \tau^{*} \leq \bar{\tau}^{-1}$ implies $\tau^{-1} \geq \bar{\tau}$. Hence, under (4.3), the matrix inequalities (4.8) are guaranteed by

$$
\left[\begin{array}{ccc}
X_{i} U_{i} X_{i} & F_{i} Y_{i} & \sqrt{\alpha_{1}+\alpha_{2}} X_{i} \\
Y_{i}^{T} F_{i}^{T} & -\alpha_{3} \rho_{i} I & 0 \\
\sqrt{\alpha_{1}+\alpha_{2}} X_{i} & 0 & -\bar{\tau} I
\end{array}\right]<0, \quad i \in S
$$

But, by the Schur complements, these MIs are equivalent to those LMIs in (4.2). The proof is therefore complete. 


\section{Stabilisation of Nonlinear Hybrid SDEs}

Let us now discuss a more general nonlinear problem. Assume that the underlying system is now described by a nonlinear hybrid SDE

$$
d x(t)=f(x(t), r(t), t) d t+g(x(t), r(t), t) d w(t) .
$$

Here, $f$ and $g$ are both mappings from $R^{n} \times S \times R_{+}$to $R^{n}$. Assume that both $f$ and $g$ are locally Lipschitz continuous and obey the linear growth condition (see e.g. [21]).

Suppose that the given SDE (5.1) is unstable and we are required to design a delay feedback control $u(x(t-\tau), r(t))$ in the drift part so that the controlled system

$$
d x(t)=[f(x(t), r(t), t)+u(x(t-\tau), r(t))] d t+g(x(t), r(t), t) d w(t)
$$

will be mean-square exponentially stable. As the given SDE (5.1) is nonlinear, we may need to design nonlinear controls. However, we here consider only a class of SDEs which can be stabilised by linear feedback controls. As in the linear case, we therefore consider the structure control of the form $u(x, i)=F(i) G(i) x$, where $F$ and $G$ are mappings from $S$ to $R^{n \times l}$ and $R^{l \times n}$, respectively, and one of them is given while the other needs to be designed. As a result, the controlled system (5.2) becomes

$$
d x(t)=[f(x(t), r(t), t)+F(r(t)) G(r(t)) x(t-\tau)] d t+g(x(t), r(t), t) d w(t) .
$$

Again, this controlled system is a hybrid SDDE. Given that our underlying equation (5.1) is non-delay and it only requires the initial value $x(0) \in R^{n}$, it is more natural to assume that for our controlled system (5.3) we know the initial data $x(t)$ on $t \in[0, \tau]$. This can be interpreted as follows: Let the underlying equation (5.1) evolve from time 0 to $\tau$ and observe the whole segment $\{x(t): 0 \leq t \leq \tau\}$. Starting from $\tau$ on, design the feedback control $F(r(t)) G(r(t)) x(t-\tau)$ based on the past observation $\{x(t): 0 \leq t \leq \tau\}$ as well as furthermore observation as time evolves. In other words, we shall regard the controlled system (5.3) as an SDDE on $t \geq \tau$ with the initial data $\{x(t): 0 \leq t \leq \tau\}$ which are generated by the $\operatorname{SDE}(5.1)$ given the initial value $x(0) \in R^{n}$. By the theory of hybrid SDEs (see e.g. [14]), we know

$$
\mathbb{E}|x(t)|^{2}<\infty \quad \text { on } t \in[0, \tau]
$$

which in turn implies, by the theory of hybrid SDDEs (see e.g. [21]), that

$$
\mathbb{E}|x(t)|^{2}<\infty \quad \text { for } t \geq \tau .
$$

Our aim is to design either $G(\cdot)$ given $F(\cdot)$ or $F(\cdot)$ given $G(\cdot)$ so that $\mathbb{E}|x(t)|^{2}$ will tend to zero exponentially.

We still use the Lyapunov functional defined by (3.1). By the Itô formula (see e.g. $[16,21])$, we have

$$
d V\left(\hat{x}_{t}, r(t), t\right)=L V\left(\hat{x}_{t}, r(t), t\right) d t+2 x^{T}(t) Q(r(t)) g(x(t), r(t), t) d w(t),
$$


for $t \geq 2 \tau$, where, when $r(t)=i$,

$$
\begin{aligned}
L V\left(\hat{x}_{t}, i, t\right) & =2 x^{T}(t) Q_{i}\left[f(x(t), i, t)+F_{i} G_{i} x(t-\tau)\right] \\
& +g^{T}(x(t), i, t) Q_{i} g(x(t), i, t)+\sum_{j=1}^{N} \gamma_{i j} x^{T}(t) Q_{j} x(t) \\
& +\alpha_{1} \tau|x(t)|^{2}-\alpha_{1} \int_{t-\tau}^{t}|x(s)|^{2} d s \\
& +\alpha_{2} \tau|x(t-\tau)|^{2}-\alpha_{2} \int_{t-\tau}^{t}|x(s-\tau)|^{2} d s .
\end{aligned}
$$

Clearly, Lemma 3.1 still holds for the non-linear controlled SDDE (5.3) with $L V$ being defined by (5.5).

Given that we use a linear control to stabilise a nonlinear system, it is natural to impose some conditions on the nonlinear coefficients $f$ and $g$. More precisely, we observe from (5.5) that we need to use the linear term $2 x^{T}(t) Q_{i} F_{i} G_{i} x(t-\tau)$ to control the nonlinear terms $2 x^{T} Q_{i} f(x, i, t)$ and $g^{T}(x(t), i, t) Q_{i} g(x(t), i, t)$. This observation leads us to impose the following assumption.

Assumption 5.1 For each $i \in S$, there is a pair of symmetric $n \times n$-matrices $Q_{i}$ and $\bar{Q}_{i}$ with $Q_{i}$ being positive-definite such that

$$
2 x^{T} Q_{i} f(x, i, t)+g^{T}(x, i, t) Q_{i} g(x, i, t) \leq x^{T} \bar{Q}_{i} x
$$

for all $(x, i, t) \in R^{n} \times S \times R_{+}$.

Moreover, we will write

$$
\begin{aligned}
& 2 x^{T}(t) Q_{i} F_{i} G_{i} x(t-\tau)=2 x^{T}(t) Q_{i} F_{i} G_{i}(x(t)-[x(t)-x(t-\tau)]) \\
& \leq 2 x^{T}(t) Q_{i} F_{i} G_{i} x(t)+\alpha_{3}^{-1}\left|x^{T}(t) Q_{i} F_{i} G_{i}\right|^{2}+\alpha_{3}|x(t)-x(t-\tau)|^{2},
\end{aligned}
$$

whence we need to estimate $\mathbb{E}|x(t)-x(t-\tau)|^{2}$. For this purpose we impose one more assumption.

Assumption 5.2 There is a pair of positive constants $\delta_{1}$ and $\delta_{2}$ such that

$$
|f(x, i, t)|^{2} \leq \delta_{1}|x|^{2} \quad \text { and } \quad|g(x, i, t)|^{2} \leq \delta_{2}|x|^{2}
$$

for all $(x, i, t) \in R^{n} \times S \times R_{+}$.

\subsection{Design $F(\cdot)$ given $G(\cdot)$}

Let us first consider the case when $G(\cdot)$ is given so we need to design $F(\cdot)$.

Theorem 5.3 Let Assumptions 5.1 and 5.2 hold. Choose five positive numbers $\alpha_{1}, \alpha_{2}, \alpha_{3}$ and $\beta_{1}, \beta_{2}$ such that

$$
\beta_{1}>2 \delta_{2}, \quad \alpha_{1}>\alpha_{3} \beta_{1}, \quad \alpha_{2}>\alpha_{3} \beta_{2}
$$


Assume that for these chosen numbers, the following LMIs

$$
\left[\begin{array}{cc}
\bar{K}_{i} & Q_{i} F_{i} G_{i} \\
G_{i}^{T} F_{i}^{T} Q_{i} & -\alpha_{3} I
\end{array}\right]<0, \quad i \in S
$$

have solutions $\bar{\tau}>0$ and $F_{i} \in R^{n \times l}$, where $I$ is the $n \times n$ identity matrix and

$$
\bar{K}_{i}=\bar{Q}_{i}+\sum_{j=1}^{N} \gamma_{i j} Q_{j}+Q_{i} F_{i} G_{i}+G_{i}^{T} F_{i}^{T} Q_{i}+\left(\alpha_{1}+\alpha_{2}\right) \bar{\tau} I .
$$

Let $\tau^{*}$ be the largest number in $(0, \bar{\tau}]$ which obeys

$$
4 \tau^{*} \delta_{1}+2 \delta_{2} \leq \beta_{1} \quad \text { and } \quad 4 \tau^{*} \max _{i \in S}\left(\left\|F_{i} G_{i}\right\|^{2}\right) \leq \beta_{2} .
$$

Then, if $\tau \leq \tau^{*}$, the controlled system (5.3) is exponentially stable in mean square.

Proof. By Assumption 5.1, we derive from (5.5) that

$$
\begin{aligned}
L V\left(\hat{x}_{t}, i, t\right) & \leq 2 x^{T}(t) \bar{Q}_{i} x(t)+2 x^{T}(t) Q_{i} F_{i} G_{i} x(t-\tau)+\sum_{j=1}^{N} \gamma_{i j} x^{T}(t) Q_{j} x(t) \\
& +\alpha_{1} \tau|x(t)|^{2}-\alpha_{1} \int_{t-\tau}^{t}|x(s)|^{2} d s \\
& +\alpha_{2} \tau|x(t-\tau)|^{2}-\alpha_{2} \int_{t-\tau}^{t}|x(s-\tau)|^{2} d s .
\end{aligned}
$$

Using (5.6) we then have

$$
\begin{aligned}
L V\left(\hat{x}_{t}, i, t\right) & \leq x^{T}(t) H_{i} x(t)-\alpha_{2} \tau\left(|x(t)|^{2}-|x(t-\tau)|^{2}\right)+\alpha_{3}|x(t)-x(t-\tau)|^{2} \\
& -\alpha_{1} \int_{t-\tau}^{t}|x(s)|^{2} d s-\alpha_{2} \int_{t-\tau}^{t}|x(s-\tau)|^{2} d s
\end{aligned}
$$

where

$$
H_{i}=K_{i}+\alpha_{3}^{-1} Q_{i} F_{i} G_{i}\left(Q_{i} F_{i} G_{i}\right)^{T},
$$

in which

$$
K_{i}=\bar{Q}_{i}+\sum_{j=1}^{N} \gamma_{i j} Q_{j}+Q_{i} F_{i} G_{i}+G_{i}^{T} F_{i}^{T} Q_{i}+\left(\alpha_{1}+\alpha_{2}\right) \tau I .
$$

However, by the well-known Schur complements, the LMIs (5.8) are equivalent to the following MIs

$$
\bar{H}_{i}:=\bar{K}_{i}+\alpha_{3}^{-1} Q_{i} F_{i} G_{i}\left(Q_{i} F_{i} G_{i}\right)^{T}<0, \quad i \in S .
$$

As $\tau \leq \bar{\tau}$, we then have

$$
H_{i}<0, \quad i \in S
$$

Noting

$$
\begin{aligned}
x(t)-x(t-\tau) & \left.=\int_{t-\tau}^{t} f(x(s), r(s), s)+F(r(s)) G(r(s)) x(s-\tau)\right] d s \\
& +\int_{t-\tau}^{t} g(x(s), r(s), s) d w(s),
\end{aligned}
$$


we estimate, by Assumption 5.2, that

$$
\begin{aligned}
\mathbb{E}|x(t)-x(t-\tau)|^{2} & \leq 2 \tau \mathbb{E} \int_{t-\tau}^{t}|f(x(s), r(s), s)+F(r(s)) G(r(s)) x(s-\tau)|^{2} d s \\
& +2 \mathbb{E} \int_{t-\tau}^{t}|g(x(s), r(s), s)|^{2} d s \\
& \leq \bar{\beta}_{1} \mathbb{E} \int_{t-\tau}^{t}|x(s)|^{2} d s+\bar{\beta}_{2} \mathbb{E} \int_{t-\tau}^{t}|x(s-\tau)|^{2} d s,
\end{aligned}
$$

where

$$
\bar{\beta}_{1}=4 \tau \delta_{1}+2 \delta_{2} \quad \text { and } \quad \bar{\beta}_{2}=4 \tau \max _{i \in S}\left(\left\|F_{i} G_{i}\right\|^{2}\right) .
$$

Recalling (5.9) we observe that $\bar{\beta}_{1} \leq \beta_{1}$ and $\bar{\beta}_{2} \leq \beta_{2}$. Set

$$
-\lambda=\max _{i \in S} \lambda_{\max }\left(H_{i}\right) .
$$

By (5.13), $\lambda>0$. Now, replacing $i$ by $r(t)$ in (5.11), taking the expectation on both sides of (5.11) and then making use the above estimations we obtain

$$
\begin{aligned}
\mathbb{E}\left(L V\left(\hat{x}_{t}, r(t), t\right)\right) & \leq-\left(\lambda+\alpha_{2} \tau\right) \mathbb{E}|x(t)|^{2}+\alpha_{2} \tau \mathbb{E}|x(t-\tau)|^{2} \\
& -\left(\alpha_{1}-\alpha_{3} \beta_{1}\right) \mathbb{E} \int_{t-\tau}^{t}|x(s)|^{2} d s \\
& -\left(\alpha_{2}-\alpha_{3} \beta_{2}\right) \mathbb{E} \int_{t-\tau}^{t}|x(s-\tau)|^{2} d s \\
& \leq-\lambda_{1} \mathbb{E}|x(t)|^{2}+\lambda_{2} \mathbb{E}|x(t-\tau)|^{2}-\lambda_{3} \mathbb{E} \int_{t-2 \tau}^{t}|x(s)|^{2} d s,
\end{aligned}
$$

where

$$
\lambda_{1}:=\left(\lambda+\alpha_{2} \tau\right)>\lambda_{2}:=\alpha_{2} \tau \quad \text { and } \quad \lambda_{3}:=\left(\alpha_{1}-\alpha_{3} \beta_{1}\right) \wedge\left(\alpha_{1}-\alpha_{3} \beta_{1}\right)>0 .
$$

Hence the conclusion follows from Lemma 3.1.

Theorem 5.3 depends on the choices of $2 N$ matrices $Q_{i}$ and $\bar{Q}_{i}$. In theory, it is flexible, but in practice, it means more work to be done in finding these $2 N$ matrices. It is in this spirit that we introduce a stronger assumption.

Assumption 5.4 There are $N+1$ symmetric $n \times n$-matrices $Z$ and $Z_{i}(i \in S)$ with $Z$ being positive-definite such that

$$
2 x^{T} Z f(x, i, t)+g^{T}(x, i, t) Z g(x, i, t) \leq x^{T} Z_{i} x
$$

for all $(x, i, t) \in R^{n} \times S \times R_{+}$.

Corollary 5.5 Let Assumptions 5.2 and 5.4 hold. Choose five positive numbers $\alpha_{1}, \alpha_{2}, \alpha_{3}$ and $\beta_{1}, \beta_{2}$ which obey (5.7). Assume that for these chosen numbers, the following LMIs

$$
\left[\begin{array}{cc}
\bar{K}_{i} & Y_{i} G_{i} \\
G_{i}^{T} Y_{i}^{T} & -\alpha_{3} I
\end{array}\right]<0, \quad i \in S
$$


have solutions $\bar{\tau}>0, q_{i}>0$ and $Y_{i} \in R^{n \times l}$, where $I$ is the $n \times n$ identity matrix and

$$
\bar{K}_{i}=q_{i} Z_{i}+\sum_{j=1}^{N} \gamma_{i j} q_{j} Z+Y_{i} G_{i}+G_{i}^{T} Y_{i}^{T}+\left(\alpha_{1}+\alpha_{2}\right) \bar{\tau} I .
$$

Let $\tau^{*}$ be the largest number in $(0, \bar{\tau}]$ which obeys

$$
4 \tau^{*} \delta_{1}+2 \delta_{2} \leq \beta_{1} \quad \text { and } \quad 4 \tau^{*} \max _{i \in S}\left(\left\|\left(q_{i} Z\right)^{-1} Y_{i} G_{i}\right\|^{2}\right) \leq \beta_{2} .
$$

Then, if $\tau \leq \tau^{*}$, by setting

$$
F_{i}=\left(q_{i} Z\right)^{-1} Y_{i}, \quad i \in S,
$$

the controlled system (5.3) is exponentially stable in mean square.

Proof. Assumption 5.4 implies

$$
2 x^{T}\left(q_{i} Z\right) f(x, i, t)+g^{T}(x, i, t)\left(q_{i} Z\right) g(x, i, t) \leq x^{T}\left(q_{i} Z_{i}\right) x .
$$

This means that Assumption 5.1 holds with $Q_{i}=q_{i} Z$ and $\bar{Q}_{i}=q_{i} Z_{i}$. Hence the corollary follows immediately from Theorem 5.3.

An even simpler (but in fact stronger) condition is:

Assumption 5.6 There are constants $z_{i}(i \in S)$ such that

$$
2 x^{T} f(x, i, t)+|g(x, i, t)|^{2} \leq z_{i}|x|^{2}
$$

for $\operatorname{all}(x, i, t) \in R^{n} \times S \times R_{+}$.

This assumption implies Assumption 5.4 with $Z=I$ and $Z_{i}=z_{i} I$. Hence, under Assumptions 5.2 and 5.6, Corollary 5.5 holds with $Z=I$ and $Z_{i}=z_{i} I$.

\subsection{Design $G(\cdot)$ given $F(\cdot)$}

Let us now consider the case when $F(\cdot)$ is given so we need to design $G(\cdot)$. The results established in the previous subsection work for this case as long as we treat $F$ as given and seek for $G$. To be precise, let us state:

Theorem 5.7 Theorem 5.3 holds for this case, if the LMIs (5.8) have solutions $\bar{\tau}>0$ and $G_{i} \in R^{l \times n}$.

Corollary 5.8 Let Assumptions 5.2 and 5.4 hold. Choose five positive numbers $\alpha_{1}, \alpha_{2}, \alpha_{3}$ and $\beta_{1}, \beta_{2}$ which obey (5.7). Assume that for these chosen numbers, the following LMIs

$$
\left[\begin{array}{cc}
\bar{K}_{i} & Z F_{i} Y_{i} \\
Y_{i}^{T} F_{i}^{T} Z & -\alpha_{3} I
\end{array}\right]<0, \quad i \in S
$$

have solutions $\bar{\tau}>0, q_{i}>0$ and $Y_{i} \in R^{l \times n}$, where $I$ is the $n \times n$ identity matrix and

$$
\bar{K}_{i}=q_{i} Z_{i}+\sum_{j=1}^{N} \gamma_{i j} q_{j} Z+Z F_{i} Y_{i}+Y_{i}^{T} F_{i}^{T} Z+\left(\alpha_{1}+\alpha_{2}\right) \bar{\tau} I .
$$

Let $\tau^{*}$ be the largest number in $(0, \bar{\tau}]$ which obeys

$$
4 \tau^{*} \delta_{1}+2 \delta_{2} \leq \beta_{1} \quad \text { and } \quad 4 \tau^{*} \max _{i \in S}\left(\left\|q_{i}^{-1} F_{i} Y_{i}\right\|^{2}\right) \leq \beta_{2} .
$$

Then, if $\tau \leq \tau^{*}$, by setting

$$
G_{i}=q_{i}^{-1} Y_{i}, \quad i \in S,
$$

the controlled system (5.3) is exponentially stable in mean square. 


\section{Examples}

Let us now discuss some examples to illustrate our theory.

Example 6.1 Consider the controlled SDDE (2.3) with the system matrices given below:

$$
\begin{gathered}
S=\{1,2\}, \quad \Gamma=\left[\begin{array}{rr}
-1 & 1 \\
1 & -1
\end{array}\right] ; \quad A_{1}=\left[\begin{array}{rr}
1 & -1 \\
1 & -5
\end{array}\right], \quad A_{2}=\left[\begin{array}{rr}
-5 & -1 \\
1 & 1
\end{array}\right] ; \\
B_{1}=\left[\begin{array}{rr}
1 & 1 \\
1 & -1
\end{array}\right], \quad B_{2}=\left[\begin{array}{rr}
-1 & -1 \\
-1 & 1
\end{array}\right] ; \quad G_{1}=(1,0), \quad G_{2}=(0,1) .
\end{gathered}
$$

Our aim here is to seek for a mapping $F: S \rightarrow R^{2 \times 1}$ and $\tau^{*}>0$ such that if $\tau \leq \tau^{*}$, then the controlled SDDE (2.3) is exponentially stable in mean square. To apply Theorem 3.2, we choose

$$
\alpha_{1}=61, \quad \alpha_{2}=101, \quad \alpha_{3}=10, \quad \beta_{1}=6, \quad \beta_{2}=10 .
$$

Noting $\left\|B_{1}\right\|^{2}=\left\|B_{2}\right\|^{2}=2$, we see that these positive numbers satisfy (3.7). It is also not difficult to verify that the LMIs (3.8) have solutions

$$
\bar{\tau}=0.02, \quad Q_{1}=\left[\begin{array}{ll}
1 & 0 \\
0 & 2
\end{array}\right], \quad Q_{2}=\left[\begin{array}{ll}
2 & 0 \\
0 & 1
\end{array}\right], \quad Y_{1}=\left[\begin{array}{c}
-10 \\
0
\end{array}\right], \quad Y_{2}=\left[\begin{array}{c}
0 \\
-10
\end{array}\right] .
$$

It is also easy to compute

$$
\left\|A_{1}\right\|^{2}=\left\|A_{2}\right\|^{2}=27.42, \quad\left\|Q_{1}^{-1} Y_{1} G_{1}\right\|^{2}=\left\|Q_{1}^{-1} Y_{1} G_{1}\right\|^{2}=100 .
$$

By $(3.9), \tau^{*}$ is the largest number in $(0,0.02]$ which obeys

$$
4 \times 27.42 \tau^{*}+4 \leq 6 \text { and } 400 \tau^{*} \leq 10,
$$

whence $\tau^{*}=0.0182$. By Theorem 3.2, if $\tau \leq 0.0182$, by setting

$$
F_{1}=\left[\begin{array}{c}
-10 \\
0
\end{array}\right], \quad F_{2}=\left[\begin{array}{c}
0 \\
-10
\end{array}\right]
$$

the corresponding controlled SDDE will be exponentially stable in mean square.

Example 6.2 Let us now discuss one more example, where we will not only illustrate our theory but also explain a new concept which may motivate a further research.

Let $r(t), t \geq 0$, be a right-continuous Markov chain on the probability space taking values in the state space $S=\{1,2\}$ with generator

$$
\Gamma=\left[\begin{array}{rr}
-\gamma_{12} & \gamma_{12} \\
\gamma_{21} & -\gamma_{21}
\end{array}\right]
$$

Consider an unstable nonlinear hybrid SDE

$$
d x(t)=f(x(t), r(t), t) d t+g(x(t), r(t), t) d w(t) .
$$

Here, $f$ and $g$ are both mappings from $R^{n} \times S \times R_{+}$to $R^{n}$. This SDE may be regarded as a system which switches between two operation modes, say mode 1 and mode 2 , and 
the switching obeys the law of the Markov chain, where in mode 1, the system evolves according to the SDE

$$
d x(t)=f(x(t), 1, t) d t+g(x(t), 1, t) d w(t)
$$

while in mode 2, according to the other SDE

$$
d x(t)=f(x(t), 2, t) d t+g(x(t), 2, t) d w(t) .
$$

Assume that in mode 1 , the state $x(t)$ can be observed completely but in mode 2 , it is not observable. Therefore, we can design a feedback control in mode 1, with some time lag of course, but we cannot have a feedback control in mode 2. In terms of mathematics, the controlled SDE is

$$
d x(t)=[f(x(t), r(t), t)+F(r(t)) G(r(t)) x(t-\tau)] d t+g(x(t), r(t), t) d w(t),
$$

where $G_{1}=I$, the $n \times n$ identity matrix but $G_{2}=0$. Given $G_{2}=0$ we can simply set $F_{2}=0$. Hence, the stabilisation problem becomes: can we find a matrix $F_{1} \in R^{n \times n}$ so that the controlled SDE (6.2) becomes exponentially stable in mean square?

To give a positive answer to the question, we assume that $f$ and $g$ obey Assumptions 5.2 and 5.6. Moreover, we assume that

$$
\gamma_{21}-z_{2} \geq 3 \gamma_{12}
$$

This means that the rate at which the system switches from the unobservable mode 2 to the observable mode 1 should be sufficiently larger than the rate from mode 1 to mode 2 . This is reasonable because the system in mode 2 is not controllable while it is controllable (hence stabilisable) in mode 1.

To apply Corollary 5.5, we need to find five positive numbers $\alpha_{1}, \alpha_{2}, \alpha_{3}$ and $\beta_{1}, \beta_{2}$ that obey

$$
\beta_{1}>2 \delta_{2}, \quad \alpha_{1}>\alpha_{3} \beta_{1}, \quad \alpha_{2}>\alpha_{3} \beta_{2},
$$

as well as to find positive numbers $\bar{\tau}, q_{1}, q_{2}$ and a matrix $Y_{1} \in R^{n \times n}$ such that

$$
\left[\begin{array}{cc}
\bar{K}_{1} & Y_{1} \\
Y_{1}^{T} & -\alpha_{3} I
\end{array}\right]<0 \quad \text { and } \quad \bar{K}_{2}<0
$$

where

$$
\bar{K}_{1}=q_{1} z_{1} I+\gamma_{12}\left(q_{2}-q_{1}\right) I+Y_{1}+Y_{1}^{T}+\left(\alpha_{1}+\alpha_{2}\right) \bar{\tau} I
$$

and

$$
\bar{K}_{2}=q_{2} z_{2} I+\gamma_{21}\left(q_{1}-q_{2}\right) I+\left(\alpha_{1}+\alpha_{2}\right) \bar{\tau} I .
$$

We first choose $\beta_{1}>2 \delta_{2}$ and $\beta_{2}>0$. Let $\alpha_{3}>0$ be a free parameter to be determined and set

$$
\alpha_{1}=\alpha_{3} \beta_{1}+1, \quad \alpha_{2}=\alpha_{3} \beta_{2}+1 .
$$

Clearly, (6.6) is therefore satisfied. Choose furthermore that

$$
\bar{\tau}=\frac{1}{3\left(\beta_{1}+\beta_{2}\right)} .
$$


Now, set

$$
q_{1}=1, \quad q_{2}=\frac{\alpha_{3}}{\gamma_{21}-z_{2}}, \quad Y_{1}=-\alpha_{3} I
$$

We then have

$$
\begin{aligned}
\bar{K}_{1} & =\left(z_{1}-\gamma_{12}+\frac{\gamma_{12} \alpha_{3}}{\gamma_{21}-z_{2}}-2 \alpha_{3}+\left[\alpha_{3}\left(\beta_{1}+\beta_{2}\right)+2\right] \bar{\tau}\right) I \\
& \leq\left(z_{1}-\gamma_{12}-\frac{4}{3} \alpha_{3}+2 \bar{\tau}\right) I
\end{aligned}
$$

and

$$
\bar{K}_{2}=\left(\gamma_{21}-\alpha_{3}+\left[\alpha_{3}\left(\beta_{1}+\beta_{2}\right)+2\right] \bar{\tau}\right) I=\left(\gamma_{21}-\frac{2}{3} \alpha_{3}+2 \bar{\tau}\right) I .
$$

We now set

$$
\alpha_{3}=\left[3\left(z_{1}-\gamma_{12}+2 \bar{\tau}+1\right)\right] \vee\left[1.5\left(\gamma_{21}+2 \bar{\tau}+1\right)\right] .
$$

Then

$$
\bar{K}_{1} \leq-\left(\alpha_{3}+1\right) I \text { and } \quad \bar{K}_{2} \leq-I \text {, }
$$

whence

$$
\left[\begin{array}{cc}
\bar{K}_{1} & Y_{1} \\
Y_{1}^{T} & -\alpha_{3} I
\end{array}\right] \leq\left[\begin{array}{cc}
-\left(\alpha_{3}+1\right) I & -\alpha_{3} I \\
-\alpha_{3} I & -\alpha_{3} I
\end{array}\right]<0
$$

namely (6.5) is satisfied. In summary, we choose $\beta_{1}>2 \delta_{2}, \beta_{2}>0$, and set $\bar{\tau}, \alpha_{3}, \alpha_{1}$ and $\alpha_{2}$ by (6.7), (6.11) and (6.6), respectively, then $\bar{\tau}$ and $q_{1}, q_{2}, Y_{1}$ specified by (6.8) obey the LMI (6.5).

Finally, let $\tau^{*}$ be the largest number in $(0, \bar{\tau}]$ that obeys

$$
4 \tau^{*} \delta_{1}+2 \delta_{2} \leq \beta_{1} \quad \text { and } 4 \tau^{*}\left(\gamma_{21}-z_{2}\right) \leq \beta_{2},
$$

namely

$$
\tau^{*}=\left(\frac{1}{3\left(\beta_{1}+\beta_{2}\right)}\right) \wedge\left(\frac{\beta_{1}-2 \delta_{2}}{4 \delta_{2}}\right) \wedge\left(\frac{\beta_{2}}{4\left(\gamma_{21}-z_{2}\right)}\right) .
$$

Then, by Corollary 5.5, we can conclude that if $\tau \leq \tau^{*}$, by setting

$$
F_{1}=-\alpha_{3} I
$$

the controlled system (6.2) is exponentially stable in mean square.

\section{$7 \quad$ Further Comments}

In this paper we have shown clearly that unstable hybrid SDEs can be stabilised by delay state feedback and output injection. Let us make a few comments to close our paper.

First of all, we emphasise once again that to make our theory more understandable as well as to avoid complicated notations, we have restricted our underlying hybrid systems to a relatively simple form, namely the hybrid SDE (1.1) driven by a scalar Brownian motion. Our theory can certainly be generalised to cope with more general hybrid SDEs driven by multi-dimensional Brownian motions as well as SDDEs.

Mathematically speaking, our stability analysis is based on the Lyapunov functional defined by (3.1). It is certainly possible to design more general Lyapunov functionals to obtain more general stabilisation criteria, for example

$$
V\left(\hat{x}_{t}, r(t), t\right)=x^{T}(t) Q(r(t)) x(t)+\int_{t-\tau}^{t} \int_{s}^{t}\left[x^{T}(u) U_{1} x(u)+x^{T}(u-\tau) U_{2} x(u-\tau)\right] d u d s,
$$


with $U_{1}=U_{1}^{T} \geq 0$ and $U_{2}=U_{2}^{T} \geq 0$.

Moreover, Example 6.2 demonstrates that it is possible to stabilise an unstable hybrid SDE even though we can only control the system in some modes. The idea illustrated there can be developed into a general partial control problem but we will report elsewhere due to the page limit of this paper.

\section{Acknowledgements}

The authors would like to thank the referees for their helpful comments and suggestions. This paper was initiated during X. Mao visited the University of Hong Kong in the early of 2007 and he wishes to thank the hospitality of the Department of Mechanical Engineering there. The authors would also like to thank the financial supports from RGC HKU 7029/05P.

\section{References}

[1] Basak, G.K., Bisi, A. and Ghosh, M.K., Stability of a random diffusion with linear drift, J. Math. Anal. Appl. 202 (1996), 604-622.

[2] Berman, A. and Plemmons, R.J., Nonnegative Matrices in the Mathematical Sciences, SIAM, 1994.

[3] Boyd, S., EI Ghaoul, L., Feron, R. and Balakrishnan, V., Linear Matrix Inequalities in System and Control Theory, SIAM, 1994.

[4] Chen, W., Guan, Z. and Lu, X., Delay-dependent exponential stability of uncertain stochastic systems with multiple delays: an LMI approach, Systems Control Lett. $\mathbf{5 4}$ (2005), 547-555.

[5] Gao, H., Wang, C. and Wang, L., On $H_{\infty}$ performance analysis for continuous-time stochastic systems with polytopic uncertainties, Circuits Systems Signal Processing 24 (2005), 415-429.

[6] Has'minskii, R.Z., Stochastic Stability of Differential Equations, Sijthoff and Noordhoff, 1981.

[7] He, H., Ho, D.W.C. and Lam, J., Stochastic stability analysis of fuzzy Hopfield neural networks with time-varying delays, IEEE Trans. Circuits and Systems II: Express Briefs $5 \mathbf{2}$ (2005), 251 -255.

[8] Ji, Y. and Chizeck, H.J., Controllability, stabilizability and continuous-time Markovian jump linear quadratic control, IEEE Trans. Automat. Control 35 (1990), 777788.

[9] Kolmanovskii, V.B. and Myshkis, A., Applied Theory of Functional Differential Equations, Kluwer Academic Publishers, 1992.

[10] Ladde, G.S. and Lakshmikantham, V., Random Differential Inequalities, Academic Press, 1980. 
[11] Mao, X., Stability of Stochastic Differential Equations with Respect to Semimartingales, Longman Scientific and Technical, 1991.

[12] Mao, X., Exponential Stability of Stochastic Differential Equations, Marcel Dekker, 1994.

[13] Mao X., Stochastic Differential Equations and Their Applications, Chichester: Horwood Pub., 1997.

[14] Mao, X., Stability of stochastic differential equations with Markovian switching, Sto. Proc. Their Appl. 79 (1999), 45-67.

[15] Mao, X., Stochastic functinal differential equations with Markovian switching, Functional Differential Equations 6(3-4) (1999), 375-396.

[16] Mao, X., Exponential stability of stochastic delay interval systems with Markovian switching, IEEE Trans. Auto. Control 47(10) (2002), 1604-1612.

[17] Mao, X., Stability and stabilization of stochastic differential delay equations, IET Control Theory \& Applications 1(6) (2007), 1551-1566.

[18] Mao, X., Lam, J., Xu, S. and Gao, H., Razumikhin method and exponential stability of hybrid stochastic delay interval systems, Journal of Mathematical Analysis and Applications 314 (2006), 45-66.

[19] Mao, X., Matasov, A. and Piunovskiy, A.B., Stochastic differential delay equations with Markovian switching, Bernoulli 6(1) (2000), 73-90.

[20] Mao, X., Yin, G. and Yuan, C., Stabilization and destabilization of hybrid systems of stochastic differential equations, Automatica 43 (2007), 264-273.

[21] Mao, X. and Yuan, C., Stochastic Differential Equations with Markovian Switching, Imperial College Press, 2006.

[22] Mariton, M., Jump Linear Systems in Automatic Control, Marcel Dekker, 1990.

[23] Mohammed, S.-E.A., Stochastic Functional Differential Equations, Longman Scientific and Technical, 1986.

[24] Niu, Y., Ho, D.W.C. and Lam, J., Robust integral sliding mode control for uncertain stochastic systems with time-varying delay, Automatica 41 (2005), 873-880.

[25] Shaikhet, L., Stability of stochastic hereditary systems with Markov switching, Theory of Stochastic Processes 2(18) (1996), 180-184.

[26] Shi, P., Mahmoud, M.S., Yi, J. and Ismail, A., Worst case control of uncertain jumping systems with multi-state and input delay information, Information Sciences 176 (2006), 186-200.

[27] Sun, M., Lam, J., Xu, S. and Zou, Y., Robust exponential stabilization for Markovian jump systems with mode-dependent input delay, Automatica 43 (2007), 1799-1807.

[28] Wei, G., Wang, Z., Shu, H. and Fang, J., Robust $H_{\infty}$ control of stochastic time-delay jumping systems with nonlinear disturbances, Optim. Control Appl. Meth. 27 (2006), $255-271$. 
[29] Xu, S. and Chen, T., $H_{\infty}$ output feedback control for uncertain stochastic systems with time-varying delays, Automatica 40 (2004), 2091-2098.

[30] Xu, S., Lam, J., Mao, X. and Y. Zou, A new LMI condition for delay-dependent robust stability of stochastic time-delay systems, Asian Journal of Control 7(4) (2005), 419-423.

[31] Xu, S., Lam, J., Wang J.L. and Yang, G.H., Stabilization and $H_{\infty}$ control for uncertain stochastic time-delay systems via non-fragile controllers, Asian Journal of Control 8 (2006), 197-200.

[32] Yue, D. and Han, Q., Delay-dependent exponential stability of stochastic systems with time-varying delay, nonlinearity, and Markovian switching, IEEE Trans. Automat. Control 50 (2005), 217-222. 